Les stratégies argumentatives dans L'Idiot de la famille

Julie Anselmini

\title{
OpenEdition
}

1 Journals

Édition électronique

URL : http://journals.openedition.org/recherchestravaux/227

DOI : 10.4000/recherchestravaux.227

ISSN : 1969-6434

Éditeur

UGA Éditions/Université Grenoble Alpes

Édition imprimée

Date de publication : 15 octobre 2007

Pagination : 93-106

ISBN : 978-2-84310-111-3

ISSN : 0151-1874

Référence électronique

Julie Anselmini, « Les stratégies argumentatives dans L'Idiot de la famille », Recherches \& Travaux [En ligne], 71 | 2007, mis en ligne le 15 avril 2009, consulté le 08 septembre 2020. URL : http://

journals.openedition.org/recherchestravaux/227 ; DOI : https://doi.org/10.4000/recherchestravaux. 227 
Julie ANSELMini

Université Grenoble 3-Stendhal

\section{Les stratégies argumentatives dans L'Idiot de la famille}

Ne me parlezpas de ce que vous dites. Je ne vous demande pas ce que vous dites. Je vous demande comment vous le dites. Cela seul est intéressant. Cela seul m'intéresse. Parlez-moi de comment vous le dites. Cela seul prouve.

Cela seul apporte et peut apporter une preuve. [...] C'était une opération, une affaire de vie, d'existence, d'être, parce que c'était l'affaire de votre propre vie, de votre propre existence, de votre propre être ${ }^{\mathrm{I}}$. Charles Péguy, Un poète l'a dit, I 907

Sartre attribue à L'Idiot de la famille, situé dans le prolongement des Questions de méthode, le but d'éprouver la validité d'une méthode. Forgée dès 1957 et fondée sur un va-et-vient entre l'universel et le singulier (avec deux mouvements complémentaires, l'analyse régressive et la synthèse progressive), celle-ci doit permettre de connaître tout ce que l'on peut « savoir d'un homme, aujourd'hui ${ }^{2} »$. Pour mettre à l'épreuve cette méthode et résoudre cette question d'épistémologie, un cas d'étude est choisi : Gustave Flaubert. L'ensemble du discours tenu sur ce dernier se présente donc comme un énoncé véridique 3 , cette véridicité devant prouver la valeur de la méthode simultanément exposée et l'extension possible de cette méthode à tout homme existant ou ayant existé. Sartre déclare dans un entretien:

I. Euvres en prose complètes, t. II, Gallimard, «Bibliothèque de la Pléiade », I988, p. 820-822.

2. Jean-Paul Sartre, L'Idiot de la famille, "Préface », Gallimard, "Bibliothèque de philosophie », p. 7. Toutes les références renvoient à cette édition, en trois volumes (I 97 I, rééd. I 988 pour les deux premiers ; I 972 , rééd. I 988 pour le dernier).

3. "je considère que "Flaubert" est [...] un ensemble de vérités sur Flaubert », dira Sartre («Sartre parle de Flaubert», Michel Sicard, Essais sur Sartre. Entretiens avec Sartre, Galilée, I989, p. I49). 
Le projet profond dans le Flaubert c'est celui de montrer qu'au fond tout est communicable et qu'on peut arriver, sans être Dieu, en étant un homme comme un autre, à comprendre parfaitement, si on a les éléments qu'il faut, un homme. Je peux prévoir Flaubert, je le connais et c'est cela mon but, pour prouver que tout homme est parfaitement connaissable pourvu qu'on utilise la méthode appropriée et qu'on ait les documents nécessaires ${ }^{4}$.

L'Idiot revêt ainsi une forte dimension argumentative, la conviction du lecteur pouvant seule asseoir la vérité d'un discours que ne viennent pas toujours authentifier des preuves irréfutables. Cette dimension est explicite dans le paratexte de l'œuvre et à l'intérieur même de celle-ci, qui contient un important champ lexical de la démonstration'. C'est à ces dispositifs argumentatifs que l'on s'intéressera ici, en examinant quelles facettes, quelles visées, quels problèmes de L'Idiot ils mettent en lumière. En considérant à la fois des procédés relevant de l'invention, de la disposition et de l'élocution et sans prétendre à l'exhaustivité -, on analysera d'abord les grands types de stratégies argumentatives déployées par Sartre, pour tâcher de découvrir, à travers ses ambiguïtés, les fins spécifiques de la rhétorique sartrienne dans L'Idiot de la famille.

\section{Convaincre à toute force}

Le premier grand type de stratégie argumentative qu'on relève dans L'Idiot est une stratégie de l'évidence: il s'agit de la part de Sartre de présenter son énoncé comme certain, à l'issue ou non d'une démonstration.

Parmi les procédés qui font naître chez le lecteur un sentiment d'évidence, on citera, à côté d'autres temps de l'indicatif, l'emploi massif du présent de l'indicatif, qui impose l'idée ou l'événement avec force, comme pleinement vraie ou pleinement actualisé. L'usage quasi permanent du présent entraîne aussi une homogénéisation entre le plan du commentaire et celui de l'objet d'étude (la vie et l'œuvre de Flaubert), de sorte que ces différents plans semblent ne plus former qu'une seule réalité. De même que le présent, la modalité assertive domine très largement, avec souvent des effets d'insistance dûs à l'emploi de la négation renforcée ("l'enfant [...] n'est rien d'autre que cette famille elle-même, en tant qu'elle est vécue par un de ses membres $\left.{ }^{6} »\right)$ ou l'emploi de l'hyperbole («Gustave ne décide jamais rien »), l’utilisation de

4. «Sur L'Idiot de la famille», Situations X, Gallimard, I976, p. Io6.

5. Voir par exemple t. I, p. 360 : «Ces états ambigus [les hébétudes du petit Gustave] sont [...] à la source même du génie de Flaubert et l'un des objectifs de ce livre est de le démontrer. »

6. IDF, t. I, p. 330 . Nous soulignons.

7. IDF, t. II, p. I 208. 
tournures restrictives qui nient toute alternative («La Vérité, pour lui, n’est qu'une croyance imposée du dehors $\left.{ }^{8} »\right)$, le recours fréquent à la dislocation ("La vérité, c'est qu'il "n’a pas d'idées" et qu'il en est conscient" »), ou à l'extraction ("C'est au sortir d'un de ces égarements, n'en doutons pas, qu'il écrit à Louise : "L'Art m'épouvante ${ }^{\mathrm{ro}}$ " $)$, à valeur emphatique, ou l'usage également fréquent de l'italique, qui met en relief et rend flagrant un élément du discours (" [Flaubert] devient par son choix et, peut-être, par une élection satanique et grandiose le martyr [...] de l'impossibilité d'être homme $\left.{ }^{1 \mathrm{I}} \gg\right)$.

Relevant d'une même stratégie de l'évidence, on citera des procédés qui appartiennent traditionnellement au roman, et qui, en fictionalisant l'énoncé, évitent sa justification. En employant le discours indirect ou direct libre et le monologue intérieur autonome ${ }^{12}$, Sartre feint ainsi d'avoir directement accès à la subjectivité de Flaubert, et il prétend « traduire dans le discours » ce qui est « indisable» par Flaubert lui-même (IDF, t. I, p. 564). Cette fictionalisation du discours, qu'on pourrait nommer une stratégie du romanesque, est une des ruses les plus efficaces de Sartre, car l'énoncé glisse insidieusement vers l'énoncé ni vrai ni faux - ou vrai et faux à la fois - qui caractérise la fiction, régie par la catégorie du vraisemblable. Or comme l'affirme Sartre lui-même, « de la fiction romanesque nul ne peut s'évader sinon en jetant le livre [...] ; le lecteur n'est plus disponible pour former par lui seul une seule image : il s'est mobilisé à produire celles qu'on lui propose et celles-là seulement » (IDF, t. II, p. I 382 ). L'Idiot de la famille se dérobe en tout cas aux exigences d'un genre strictement défini pour s'installer dans un domaine incertain, sans codification stricte ni pacte de lecture clairement défini. Le jugement du lecteur est en quelque sorte suspendu, comme le note Claude Burgelin, non sans malaise : "J'admire [...], mais je me sens exclu d'un statut critique de lecteur ${ }^{13}$.»

Parmi les procédés visant à rendre évident le discours tenu sur Flaubert, on relèvera aussi une stratégie plus indirecte, qui n'impose pas immédiatement l'évidence mais la construit par des effets de composition, grâce à l'usage de la prolepse, de l'analepse et de l'anaphore.

Par un effet d'anticipation, Sartre introduit fréquemment une idée tout en remettant son développement à plus tard : «Gustave est femme, nous verrons pourquoi », dit-il par exemple dans le premier tome de L'Idiot (p. 445). Restée

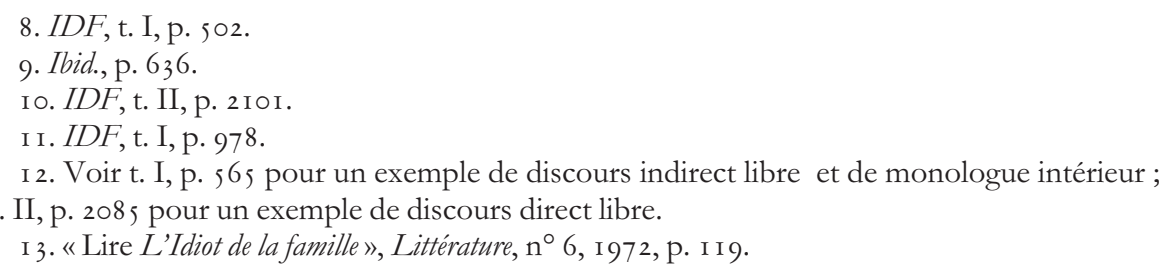


dans la mémoire du lecteur, l'idée, lorsqu'elle revient au premier plan, s'impose alors facilement, comme faisant écho à quelque chose de déjà connu. De même, l'annonce systématique des conclusions de chaque sous-partie du discours, ensuite pré-synthétisées par les sous-titres, contribue efficacement à la persuasion (d'autant plus que Sartre use abondamment d'interro-négatives à valeur oratoire $\left.{ }^{14}\right)$.

Inversement, il arrive souvent qu'une idée d'abord développée sous forme d'hypothèse soit rappelée sous forme de certitude, et devienne à son tour une grille d'interprétation et le fondement de nouvelles hypothèses. Ainsi, l'absence d'amour maternel est d'abord présenté comme une supposition, et même comme une "fable » (voir t. I, p. I38); mais il est ensuite affirmé : "C'est le zèle pieux et glacial de sa mère qui a constitué Gustave comme agent passif ; Mme Flaubert est à l'origine de cette "nature" et du malaise à travers lequel celle-ci se fait vivre.» (IDF, t. I, p. I 79) ; «il n’use jamais de sa voix pour raisonner, il s'exhibe en elle comme passivité constituée. [...] Nous savons pourquoi », déclare Sartre plus loin, érigeant l'hypothèse primitive au rang de postulat (IDF, t. I, p. 87I). Des expressions telles que « Nous ne sommes plus dupes de ", "On pouvait s'y attendre ", " Nous commençons à connaittre Flaubert et nous savons désormais que », «Nous connaissons la musique » ou «On reconnaît les méthodes de Gustave » sont familières à Sartre. Il établit par là une forte connivence avec son lecteur, dont il fait une sorte d'initié à une vérité réservée à ceux qui ont lu son livre dès le commencement, et qui ont eu le courage de le suivre. À ces initiés, une fois qu'ils ont pénétré le système de l'œuvre, il n'est presque plus besoin d'expliciter la démonstration : «Est-il besoin de noter ici la révolution qui s'est faite chez l'enfant? ? (IDF, t. II, p. II95).

La puissante mécanique de L'Idiot, avec ses introductions et ses récapitulations systématiques, ses constants phénomènes d'annonce ou de rappel, amène ainsi un ressassement, un martèlement des idées, qui les ancrent dans l'esprit du lecteur et en font son horizon obligatoire - ce qui peut être ressenti comme asphyxiant ${ }^{15}$.

Relevant également d'un phénomène de composition, on notera enfin, concernant l'ensemble de L'Idiot, la subordination de la chronologie à la dialectique, telle que Philippe Lejeune l'a mise en évidence à propos des Mots

I4. Voir par exemple t. II, p. 2025 : «N'y aurait-il pas, chez lui, deux “Qui perd gagne” [...] ? C'est ce que nous allons tenter d'établir...».

I 5 . Claude Burgelin voit ainsi dans L'Idiot une "parole étouffante qui enferme dans un vocabulaire et des formules qui [...] finissent par ne plus renvoyer qu'à elles-mêmes » («Lire L'Idiot de la famille », art. cité, p. I I9), " un discours ingénument totalitaire » ("De Sartre à Flaubert ou la genèse d'un roman vrai », RHLF, LXXXI-4/5, I 98 I, p. 695). 
dans Le Pacte autobiographique. Comme dans Les Mots, on assiste à une « dictature de la dialectique » d'autant plus efficace que « les articulations dialectiques [sont] représentées comme des schémas de succession historique ${ }^{16} \gg$. Pour seul exemple de cette « dictature du sens », on mentionnera le coup de force initial : en se fondant sur un texte problématique de la nièce de Flaubert, Sartre part d'un fait remontant aux sept ans de l'écrivain (son rapport faussé à l'environnement familial et sa constitution pathétique déterminent sa mauvaise insertion dans le langage), puis il écrit ce qui précède comme ce qui suit dans la vie de Flaubert à la lumière de ce fait. Les déclarations de la préface cachent et dévoilent par leur désinvolture l'arbitraire fondamental de ce geste : « À présent, il faut commencer. Comment? Par quoi ? Cela importe peu : on entre dans un mort comme dans un moulin » (IDF, t. I, p. 8). Mais comme le remarque Philippe Lejeune, «le moulin [...] : c'est l'ordre d'un engrenage ${ }^{17}{ }^{17}$.

Cette dictature du sens, d'une part, aboutit à donner une structure fortement dramatique au récit, qui déploie toutes les étapes d'un projet; d'autre part, le lecteur est fait prisonnier d'un système entièrement signifiant, que ce soit au niveau de la phrase, dont le style dégage l'intentionnalité sous-jacente au moindre geste de Flaubert, ou au niveau global du livre, " grâce à l'articulation dialectique de toutes les intentions ainsi dégagées ${ }^{18} »$. C'est le paradoxe de cet ouvrage, note Jacques Lecarme à propos de ce " grand carrousel dialectique », " que, tout en dénonçant inlassablement la passivité flaubertienne, il impose à son lecteur le choix entre l'admiration passive et le renoncement pur et simple ${ }^{19} »$.

Tout un ensemble de procédés vise ainsi à imposer les idées de Sartre au lecteur. Or ces procédés sont d'autant plus efficaces que parallèlement, sont exhibées des procédures de contrôle et de validation du discours, qui affichent la scientificité de celui-ci et forgent de l'auteur un ethos rigoureux et loyal propre à convaincre le lecteur.

La rigueur scientifique du discours est d'abord construite par l'accumulation de preuves intervenant à certains endroits de la démonstration : dans le premier tome de L'Idiot, pour établir le rapport de vassalité entre Laporte et Flaubert, on trouve une pléthore de citations extraites de leur correspondance (p. 74I-742); plus loin, sont également cités de très nombreux extraits de lettres de Le Poittevin, montrant la rareté de ces lettres (IDF, t. I, p. I05 2-I054)

I6. «L’ordre du récit dans Les Mots de Sartre », Le Pacte autobiographique, Éditions du Seuil, «Poétique», 1975, p. 227.

17. Ibid., p. 229.

18. Ibid., p. 232.

19. « Sartre et son double», NRF, n 232, 1972, p. 84. 
- au contraire, à d'autres endroits, les preuves font totalement défaut ${ }^{20}$. Les auto-réfutations de Sartre, la simulation d'une contre-argumentation et le dialogisme introduits dans son discours ${ }^{21}$ ont le même effet, ainsi que la numérotation des idées ${ }^{22}$, les digressions analytiques (comme le détour par la névrose objective dans le troisième tome), les exemples ${ }^{23}$ ou le rappel périodique de la méthode et des conclusions. Exhibant la rationalité du discours, ces procédés dotent l'auteur d'un ethos méthodique et philosophique qui s'étend par contamination à l'ensemble du discours.

La loyauté de l'argumentateur est également construite grâce à l'aveu de certaines suppositions : « je crois », «j'imagine», «j'incline à penser»; « il se peut $[\ldots]$ - je n'en sais rien - ... », écrit quelquefois Sartre, qui avoue de manière encore plus nette, à propos des rapports entre Le Poittevin et sa mère : " [cette conjoncture] est indémontrable et $[. .$.$] je la donne pour ce$ qu'elle vaut» (IDF, t. I, p. IO28). Courante au début de l'œuvre, puis de plus en plus rare, cette prudence ostentatoire, également marquée par la modalisation épistémique du discours ou par les tournures hypothétiques, tend naturellement à conquérir la confiance du lecteur, de même que la posture d'enquêteur circonspect, suivant patiemment son fil d'Ariane, parfois affichée par Sartre ${ }^{24}$.

Les hypothèses émises n'en restent pas moins valables dans la démarche expérimentale de Sartre, où l'imagination est érigée en appoint heuristique. Il s'agit, comme il est dit dans les notes pour le dernier livre de L'Idiot, d'« inventer le réel» (IDF, t. III, p. 8ı o), c'est-à-dire de dire indirectement le réel par le détour de l'invention (c'est dans cette perspective qu'on peut entendre l'expression de « roman $\operatorname{vrai}^{25}$ » employée par Sartre) : "J'ai tenté d'écrire avec mon imagination aussi bien qu'avec ma raison, mais parce que je pense que l'imagination est fournisseuse de vérités, au niveau des

20. Voir t. I, p. I022 : « Nous nous contenterons [...] d'indiquer les directions dans lesquelles on aurait pu mener l'enquête si les circonstances s'étaient montrées plus favorables [si nous avions eu des informations suffisantes]. »

21. Voir par exemple t. I, p. 855 : «Gustave, se faisant acteur pour récupérer son être, est amené à jouer dans des pièces que d'autres ont écrites, son rêve de vassalité y trouve son compte; mais vers la même époque, il lui arrive aussi d'écrire des pièces pour les jouer. Cette conduite n'est-elle pas le contraire de la soumission?»

22. Voir par exemple t. I, p. 876-883 : Sartre énumère trois conséquences de la « vocation contrariée » de Flaubert.

23. Nous renvoyons sur ce point à l'article de Marielle Macé : " "Penser par cas" : pratiques de l'exemple et narration dans L'Idiot de la famille».

24. Voir t. I, p. 59 : «cette enquête nous ramène à la persona de la mère », et t. II, p. I 256 : «il faut quitter pour un moment le thème de la générosité et suivre cet autre fil d'Ariane, la dérision ».

25. «Sur L'Idiot de la famille», Situations X, éd. citée, p. 94. 
structures ${ }^{26} »$, explique Sartre dans un entretien. En outre, ce sont moins les conclusions que la méthode qui compte ${ }^{27}$. L'auteur déclare à propos des rapports de Mme Flaubert avec son nourrisson :

Je l'avoue, c'est une fable. Rien ne prouve qu'il en fut ainsi. [...] N'importe [...] : l'explication réelle, je peux m'imaginer, sans le moindre dépit, qu'elle soit exactement le contraire de celle que j'invente ; de toute manière il faudra qu'elle passe par les chemins que j'indique et qu'elle vienne réfuter la mienne sur le terrain que j'ai défini... (IDF, t. I, p. I 38$)$

Tout en se justifiant, Sartre nous révèle que sa démonstration est faillée et que l'on est, selon le mot de Jacques Lecarme, face à de la critique-fiction ${ }^{28}$. Dès lors, on peut se demander si la confiance que l'auteur demande au lecteur de lui accorder n'est pas surtout fondée sur un ethos construit en amont de L'Idiot par son œuvre antérieure et par sa célébrité : les références de Sartre à ses précédents écrits ont pour fonction d'asseoir l'ensemble du discours sur une autorité qu'il n'a plus à prouver.

Cette autorité, qui permet à l'auteur de ne pas justifier systématiquement ce qu'il avance et qui sous-tend la stratégie de l'évidence dont on a parlé, se traduit également par les nombreuses formules de L'Idiot de la famille. Employant le présent gnomique, elles ne laissent aucune prise à la réfutation : «Quand les pères ont des projets, les enfants ont des destins » (IDF, t. I, p. I 66). Bien plus, elles sont volontiers paradoxales et provocatrices (la doxa étant l'admiration commune pour Flaubert); Sartre fait fréquemment de "l'épate », comme lorsqu'il évoque « Flaubert, l'enfant préfabriqué » (IDF, t. I, p. 390) ou écrit qu'il est un «forcené du négatif» (IDF, t. I, p. 428). Le travail du style vise à imposer l'opacité signifiante des mots et de leurs agencements : quand Sartre parle à propos d'Achille-Cléophas de «puritanisme de l'utilité » (IDF, t. I, p. 76) ou de « stalinisme privé » (IDF, t. I, p. 97), la persuasion du lecteur est confiée au choc des mots. Quand il évoque le « harnais de l'imaginaire » (IDF, t. II, p. I464), c'est l'image qui remporte l'adhésion. La concision et le raccourci sont en eux-mêmes saisissants, tout en disant le plus vite et le mieux possible un ensemble de choses, ce que Sartre nomme faire de la philosophie ${ }^{29}$.

26. «Sartre parle de Flaubert», Michel Sicard, Essais sur Sartre. Entretiens avec Sartre, éd. citée, p. I48-I 49 .

27. «L'essentiel est une méthode», dit Sartre dans «Sur L'Idiot de la famille» (Situations X, éd. citée, p. 104).

28. «Sartre et son double», art. cité, p. 85.

29. Voir « Sartre parle de Flaubert», Michel Sicard, Essais sur Sartre. Entretiens avec Sartre, éd. citée, p. I 52 : « c'était ça, pour moi, la philosophie : essayer de résumer et de dire le plus vite possible - et le mieux - un ensemble de choses...» 
C'est également à des fins de sidération que Sartre utilise le registre familier : «Mme Flaubert l'avait pondu mais elle avait oublié de lui donner son visa » $(I D F$, t. I, p. 337). Cette familiarité, qui crée une forte proximité avec le lecteur, est aussi une manière de couper court à la controverse : «Croit-on qu'il ne sent pas cela, Gustave ? Parbleu, si...» (IDF, t. II, p. I 25 I).

Tout en cherchant toujours à impliquer fortement son lecteur dans sa démarche (Sartre use abondamment des fonctions phatique et surtout conative du langage), l'écrivain renonce parfois à bousculer son lecteur pour mieux le séduire - ce qui, selon Ricœur, est une des « formes les plus subtiles de la violence $^{30} »$. Pour cela, l'humour est une arme fréquemment brandie : «Il l'aimait avec fureur, soit. Mais de loin », dit Sartre à propos de Louise Colet dans le premier tome (p. 706). Le recours à un style imitant le langage parlé tend également à établir un courant de sympathie avec le lecteur. Méfiant devant la littérature ${ }^{31}$, Sartre pense de manière générale qu'« on doit écrire comme on parle ${ }^{32}$ »; le langage naturel qu'il emploie, qui se veut « un équivalent écrit de la séduction orale » (IDF, t. I, p. 889), permet de nouer une plaisante conversation avec le lecteur, et, dans la mesure où il s'oppose à l'habileté raffinée du discours sophistique, il fonctionne comme un « indice de probité ».

Le style sartrien rejoint ainsi l'idéal recherché par Montaigne, qui aime « un parler simple et naïf, tel sur le papier qu'à la bouche ; un parler succulent et nerveux, court et serré [...], plutôt difficile qu'ennuyeux ${ }^{33}$ ». Ce style péremptoire et hardi, en émerveillant et parfois en choquant le lecteur, court-circuite les voies classiques de l'argumentation.

\section{Trucage et vérité : les fins de la rhétorique}

Ces différents procédés aboutissent à créer une forte connivence avec le lecteur, et l'on notera que Sartre cherche fréquemment à tourner cette complicité contre Flaubert : l'humour se change alors en ironie et la rhétorique, de délibérative, devient judiciaire. Cette dimension polémique est sensible à travers le thème récurrent de l'insincérité flaubertienne, déclinée en falsification, hypocrisie, manipulation, mauvaise foi, mensonge, simulation, tricherie, trucage, etc. Des accusations de méchanceté ou de sournoiserie sont également

30. La Métaphore vive, Éditions du Seuil, i 975 , p. i6.

3 I. Voir La Nausée, Gallimard, I 938 . Rééd. « folio », I983, p. 85 : «Se méfier de la littérature. Il faut écrire au courant de la plume ; sans chercher les mots. »

32. Lettre du ıo mai I 940 à Simone de Beauvoir, Lettres au Castor, t. II, Gallimard, I983, p. 2 I 8.

33. Essais, t. I, p. 26. 
émises contre Flaubert, qui sont susceptibles d'orienter la démonstration. Par exemple, pour justifier le fait que Gustave ait pris la défense du censeur de son collège, en 1835 , Sartre écarte l'explication de la bonté d'âme ou de l'amour de la vérité, et conclut en postulant la passivité et le caractère « rancuneux » de Gustave (IDF, t. II, p. I 366). Le rejet par Sartre du " parti pris fixiste » de Flaubert (IDF, t. II, p. I35 I), de son anti-humanisme, de son masochisme sadique, etc., est tout aussi manifeste ; la condamnation transparaît dans les tournures restrictives que l'on a déjà mentionnées (la pensée de Flaubert «n'est que la forme verbale du pathos ${ }^{34}$ »), ou dans une compassion qui masque à peine la férocité : «Un ambitieux passif, quoi de plus misérable ?» $(I D F$, t. I, p. 353). Pour se faire du lecteur un allié plus sûr contre Flaubert, Sartre feint parfois de lui attribuer son propre énervement : «On s'agacera sans doute de cette fatuité, de cette réserve affectée ", dit-il à propos d'une lettre à Le Poittevin (IDF, t. I, p. IoIG).

Plutôt qu'un exemple destiné à montrer la validité d'une méthode, Flaubert apparaît donc souvent comme un contre-exemple en matière d'existence, ou, selon le mot de Jean-François Louette, comme un " contrehomme ${ }^{35}$ "; la rhétorique de L'Idiot de la famille semble autant au service d'une démonstration à faire que d'un contentieux à régler, contentieux que l'auteur, dans sa préface, avoue être au fondement de l'entreprise épistémique : «j’ai eu le sentiment d'un compte à régler avec lui et que je devais en vue de cela, mieux le connaître » (IDF, t. I, p. 8). Sartre reconnaît ainsi la dimension polémique de son œuvre, tout en niant qu'elle vise la personne de Flaubert : « Il y a incontestablement dans mon livre une attaque constante contre la bourgeoisie de l'époque, dont la famille Flaubert est très représentative ${ }^{36} »$, lit-on dans Situations $X$.

La rhétorique de L'Idiot apparait donc retorse : tout en revêtant l'apparence d'un discours scientifique, elle recourt à d'innombrables coups de force pour s'assurer le concours du lecteur dans une entreprise dont la visée même est ambiguë. Devant les ruses de ce discours, la tentation est grande d'instruire le procès de Sartre. On peut en effet décrier la manipulation du lecteur à laquelle il se livre, et s'insurger devant la partialité qu'il montre à l'égard de Flaubert. On peut même contester toute valeur à son propos en montrant l'approximation de sa démonstration, fondée sur des postulats discutables (par exemple, l'idée que Flaubert s'incarne directement dans ses personnages); on peut aussi

34. IDF, t. I, p. 645 .

35. Voir Jean-Paul Sartre, Hachette, « Portraits littéraires », I 993, p. 54.

36. «Sur L'Idiot de la famille», Situations X, éd. citée, p. 97. 
mettre en cause l'utilisation que fait Sartre des différents documents : non seulement l'œuvre littéraire, à propos de laquelle d'aucuns signalent des erreurs d'interprétation ${ }^{37}$, mais aussi la correspondance, où Sartre voit tantôt des confessions directes, tantôt des dénégations à lire au rebours de leur message explicite ; les méthodes critiques de Sartre elles-mêmes, inspirées notamment de la psychanalyse (que Sartre, d'après Claude Burgelin, comprend mal ${ }^{38}$ ) et du marxisme, peuvent être discutées.

Cependant, dresser un tel réquisitoire aboutit à caractériser L'Idiot de manière toute négative, et revient à instruire le vieux procès de la rhétorique, qui part de prémisses non nécessairement prouvées, qui est, contrairement à la démonstration des sciences exactes, une quasi-logique, qui est d'abord une influence intersubjective, qui revêt presque toujours une dimension polémique et qui mêle, de façon inextricable, l'affectif au rationnel, l'inextricable faisant précisément sa force ${ }^{39}$. Il semble donc vain de tomber dans ce faux débat.

Une autre piste qu'on suivrait sans beaucoup de profit consisterait à s'engager dans un débat sans fin sur le genre dont relève L'Idiot - est-ce un roman, une biographie, une fiction biographique, un traité philosophique, une mythographie, etc. ? D'abord, en effet, le genre de l'essai, caractérisé par un brouillage des frontières entre l'argumentatif et le narratif, le délibératif et le polémique, le fictionnel et le factuel, l'objectif et le subjectif ${ }^{4 \circ}$, semble tout à fait apte à rendre compte du statut de L'Idiot et de ses ambiguïtés. Ensuite, vouloir établir des lignes de démarcation trop strictes serait méconnaître la démarche propre de Sartre, qui revendique un type d'accès particulier à la vérité et se détourne de l'enquête universitaire traditionnelle pour « interroger les fantasmes de Flaubert en acceptant de se laisser porter par ses propres fantasmes $^{41} »$. Enfin, l'incertitude générique et le glissement vers la fiction sont, comme on l'a suggéré, une des stratégies les plus efficaces du texte en matière de persuasion.

Alors comment caractériser de manière positive L'Idiot de la famille d'après les stratégies rhétoriques qu'il déploie, et éclairer grâce à elles la spécificité de cette œuvre ? On trouvera un élément de réponse en se tournant non plus

37. Marthe Robert en signale une à propos de la lecture sartrienne du cauchemar rapporté dans les Mémoires d'un fou (Voir «En haine du roman », Roman des origines, origines du roman, Gallimard, i 972. «Tel », I 997, p. 3 18-319).

38. Voir «De Sartre à Flaubert ou la genèse d'un roman vrai », art. cité, p. 695 : «Là où le bât blesse le plus cruellement dans L'Idiot de la famille, c'est du côté de l'explication psychanalytique. »

39. Voir Olivier Reboul, La Rhétorique, PUF, «Que sais-je ?», I996, p. I I 9.

40. Nous renvoyons sur ce point à l'ouvrage de Pierre Glaudes et Jean-François Louette, L'Essai, Hachette, «Contours littéraires », I 999.

4I. Claude Burgelin, «De Sartre à Flaubert ou la genèse d'un roman vrai», art. cité, p. 696. 
vers les ambiguités inhérentes au genre du discours (rhétorique et essayiste), mais vers l'ambivalence du rapport entre l'auteur et son objet, et vers l'incertitude relative au véritable objet du discours.

L'examen des stratégies argumentatives a montré comment un ensemble de procédés visait à imposer comme évident le discours tenu sur Flaubert, en donnant le sentiment d'une proximité très grande, d'une sorte de fusion de Sartre avec son objet (on peut également dire qu'ils supposent et traduisent cette proximité). Le discours indirect libre, notamment, brouille la frontière entre l'auteur et son objet d'étude, au point que Sartre est parfois obligé de préciser, en note : «Je dis ce que pense Flaubert [...] ; non ce que je pense » $(I D F$, t. II, p. I 282), ou encore : « Il va de soi que nous laissons à Gustave l'entière responsabilité de ces conceptions » (IDF, t. II, p. I976) - mais s'il est besoin de préciser un tel point, c'est justement qu'il ne va pas de soi!

Sartre lui-même donne une clef pour comprendre cette proximité, cette fusion, à travers le statut qu'il attribue à la connaissance. Pour lui, celle-ci est moins un savoir qu'une compréhension, et l'empathie est la « seule attitude requise pour comprendre ${ }^{42} »$; elle seule permet de restituer une vie dans sa «saveur immédiate » (IDF, t. I, p. 394). Cette proximité est donc également une exigence du genre biographique ${ }^{43}$, qui requiert une empathie pouvant aller jusqu'à une vampirisation du biographé par son biographe - ou du biographe par son biographé (on se souvient de ce qui se passe entre Roquentin et le marquis de Rollebon dans La Nauséét4).

Selon plusieurs critiques, la fusion de l'auteur avec son objet est favorisée, dans le cas de L'Idiot de la famille, par les parentés entre la vie de Sartre et celle de Flaubert (ou celle qu'il attribue à Flaubert), notamment entre leurs deux enfances. «On retrouve en Gustave l'enfant des Mots», déclare Josette Pacaly, qui établit d'innombrables rapprochements entre les deux textes et parle d'une «identification du biographe à son objet ${ }^{45}$ ». Quant à Michel Sicard, il parle de « transfert ${ }^{46} »$ ou d'« auto-analyse ${ }^{47} »$. Bruno Clément, lui, de manière

42. IDF, « Préface», t. I, p. 8.

43. Voir IDF, t. I, p. 658 : « cette saveur qui se donne immédiatement, c'est cela même qu'il faudrait restituer au terme d'une longue fréquentation ou d'une étude biographique. »

44. Voir La Nausée, éd. citée, p. i 40 : «Moi, je fournissais la matière brute, cette matière brute dont j'avais à revendre, dont je ne savais que faire : l'existence, mon existence. Lui, sa partie, c'était de représenter. Il se tenait en face de moi, et s'était emparé de ma vie pour me représenter la sienne. [...] Je n'étais qu'un moyen de le faire vivre, il était ma raison d'être, il m'avait délivré de moi. »

45. « Sartre et son double», Sartre au miroir, Klincksieck, I 980 , p. 3 I 5 .

46. La Critique littéraire de Jean-Paul Sartre, I - objets et thèmes, Minard, « Archives des Lettres modernes ", I976, p. 42.

47. «Le continent Flaubert », Essais sur Sartre, Gallimard, I980, p. 60. 
plus large et peut-être plus nuancée, voit dans L'Idiot une énarration, un récitcommentaire, qu'il faut lire comme une dernière tentative de Sartre pour approcher à travers Flaubert la compréhension de soi qu'il a poursuivie à travers toute son œuvre, et qu'il ne peut atteindre que par la médiation de l'Autre ${ }^{4}$. C'est à ce commentaire que Sartre s'en remet " pour dire le dernier mot de ce qu'il a toute sa vie cherché à approcher sous tant de formes : la liberté, l'écriture », et pour s'affronter poétiquement aux « apories de l'identité49 ». L'Idiot serait donc une vaste confidence indirecte de Sartre sur luimême, devant être lue à deux niveaux, comme une allégorie (le discours sur Flaubert cachant systématiquement un discours sur soi).

Cette hypothèse d'une "identité énarrative » de Sartreso (il s'agit d'une identité forgée dans la confrontation avec autrui et l'œuvre d'autrui) avait déjà été suggérée par Jeanne Bem ${ }^{51}$ ou par Jean-François Louette, qui écrit dans son Jean-Paul Sartre: «Sartre aime à ne s'expliquer qu'à travers ceux dont, critique ou essayiste, il parle ${ }^{\varsigma^{2}} »$. Cette hypothèse est par ailleurs validée par Sartre lui-même, qui pense que "l'autobiographie n'est qu'une simulation ${ }^{3}$ », et qui déclare dans le Saint Genet: «On ne coïncide pas avec soi-même sans médiation ${ }^{54} »$ ou encore, dans $L ' \hat{E}$ tre et le Néant: « autrui est le médiateur indispensable entre moi et moi-mêmess ». Sa conception du style comme feuilleté, pluriel, polysémique, va également dans le sens d'une interprétation allégorique et d'une lecture à plusieurs niveaux. On lit en effet dans Situations : «Pour moi, le style (qui n'exclut pas la simplicité, au contraire) est d'abord une manière de dire trois ou quatre choses en une. Il y a la phrase simple, avec son sens immédiat, et puis, dessous, simultanément, des sens différents qui s'ordonnent en profondeur ${ }^{56}$. « «[Q]uand on écrit, on se déguise ${ }^{57}$ », déclare enfin Sartre dans une interview, avouant la part de trucage inhérente à sa propre pratique littéraire.

48. «En haine de soi », Le Lecteur et son modèle, PUF, «Écriture », I 999, p. I75-255.

49. Ibid., p. 176 et I 85 .

50. Bruno Clément forge ce concept en référence à l’identité narrative de Paul Ricœur, dans Temps et récit.

5 I. Voir «La production du sens chez Flaubert : la contribution de Sartre », La Production $d u$ sens chez Flaubert (colloque de Cerisy), U.G.E., « Io/ I 8 », I 975, p. I 57 : « Lire L'Idiot, c'est lire un roman autobiographique enfin réussi parce que l'autobiographe a eu la bonne idée de changer d'identité».

52. Jean-Paul Sartre, Hachette, «Portraits littéraires », I 993, p. I7 I.

53. «Je-Tu-Il», Situations IX, Gallimard, I 972 , p. 302-303.

54. Saint Genet comédien et martyr, Gallimard, 1952, p. 90.

55. L'Étre et le Néant, Gallimard, I 943. Édition «Tel», I 976 , p. 260.

56. «Autoportrait à soixante-dix ans », Situations X, éd. citée, p. I 37-1 38.

57. «Sur L'Idiot de la famille», Situations X, éd. citée, p. Io5. 
Dès lors, on est en droit de rapporter à Sartre lui-même les mécanismes qu'il attribue à Flaubert : celui-ci cherche à « rendre indirectement l'indisable » (IDF, t. II, p. I 200) en « charg[eant] ses personnages de matérialiser et d'individualiser $[. .$.$] ce qu'il y a d'inarticulable dans son désir fondamental » (IDF,$ t. I, p. 958); il construit ses personnages en se construisant (IDF, t. I, p. 968), et quand il « décrit de l'intérieur ses personnages », «c'est toujours, d'une certaine manière, de lui qu'il parle ${ }^{\varsigma 8} »$. Or Sartre fait de Flaubert son personnage en acceptant qu'on considère L'Idiot de la famille comme un roman's, de sorte que l'hypothèse de lecture de cette œuvre comme une réflexion indirecte de Sartre sur lui-même semble parfaitement authentifiée.

Avant d'en finir avec ce détour, on rappellera que parallèlement à cette fusion (cette confusion ?) de Sartre avec son objet, l'étude des stratégies rhétoriques a révélé une forte mise à distance, marquée par l'ironie exercée contre Flaubert, par les jugements de valeur émis contre lui, et par la construction par Sartre d'un ethos fortement individualisé, visant à séduire le lecteur et à nouer une étroite complicité avec lui, parfois aux dépens de Flaubert. Oscillant entre l'admiration pour l'œuvre et une condamnation qui n'exclut ni une forme de tendresse ${ }^{60}$, ni la fascination - à preuve, la seule entreprise, titanesque, de L'Idiot de la famille -, le rapport de Sartre avec son objet est foncièrement ambivalent.

Pour conclure sur le rôle des stratégies argumentatives dans L'Idiot de la famille, on suggérera (en retenant comme centrale l'hypothèse de Bruno Clément) que ce sont ses structures rhétoriques qui assurent à l'œuvre son bon fonctionnement et son difficile équilibre. D'un côté, en effet, elles trahissent la transparence recherchée et ressentie entre Sartre et Flaubert, et d'un autre côté, elles troublent cette transparence et contrecarrent cette fusion par l'interposition d'un ethos sartrien nettement différencié, et par l'établissement d'une relation très forte entre Sartre et son lecteur, dont il se fait un allié contre Flaubert. En affirmant vigoureusement la personnalité de l'auteur visà-vis du lecteur, les structures rhétoriques du discours le démarquent de son objet et lui permettent de ne pas se dissoudre en lui. À côté de leur rôle proprement rhétorique de persuasion, elles assument donc une fonction beuristique: à un premier niveau, elles maintiennent une distance nécessaire à la connaissance de l'objet, tout en traduisant l'empathie également nécessaire à cette

58. «Sur moi-même», Situations $I X$, éd. citée, p. I I 7 .

59. Ibid., p. I 23.

6o. "La vanité de Gustave, toute de naïveté, est émouvante ", dit ainsi Sartre dans le deuxième tome de L'Idiot (p. I 260). 
connaissance ; à un second niveau, elles fondent la tentative de compréhension et d'acceptation de soi puisqu'elles permettent de résister au vertige de l'identification tout en y cédant, rendant possible cette tentative d'« avoisinement » ou d'affleurement de soi à travers autrui que constitue L'Idiot. Enfin, les stratégies rhétoriques ont une fonction de dévoilement. D'une part, en proclamant la distance et l'altérité de Sartre par rapport à Flaubert, elles masquent leur gémellité imaginaire. Mais d'autre part, elles donnent les clefs de la confession oblique que constitue L'Idiot et révèlent Sartre derrière Flaubert, puisque l'argumentation, ostensiblement truquée (elle avoue sa part de fiction et relaie superbement l'analyse par l'audace), vise à s'auto-dénoncer. Entre transparence et opacité, les structures rhétoriques montrent le masque du doigt, tout en demeurant le dernier rempart, l'ultime recours contre la mise à nu de soi. 